\title{
Seasonal species diversity and abundance of phytoplankton from the southwestern Caspian Sea
}

\author{
N. Heydari $[$ - S. M. R. Fatemi · A. Mashinchian · R. Musavi Nadushan • \\ B. Raeisi
}

Received: 10 June 2018/Accepted: 4 December 2018/Published online: 21 December 2018

(C) The Author(s) 2018

\begin{abstract}
In the present investigation, we aimed to study diversity and cell abundance of phytoplankton in the southwest of the Caspian Sea. This survey included 4 transects and 12 stations. A total of 48 samples were collected during spring 2012 and spring 2013. Finally, 72 species of phytoplankton were identified including phyla Diatoms (29 species), Chlorophyta (17 species), Pyrrophyta (16 species), Cyanophyta (6 species), and Euglenophyta ( 4 species). The total average of cell abundance was approximately $43.55 \pm 5.10 \times 104$ cells/ $\mathrm{m}^{3}$. The number of recorded species in spring, summer, autumn, and winter was 46, 50, 41 and 39, respectively. Diatoms had the highest species diversity and cell abundance in winter related to Thalassionema nitzschiodes at a depth of $10 \mathrm{~m}$. Seasonal changes in diversity of phytoplankton significantly differed, showing maximum in autumn with high diversity index (2.532) and minimum in spring (2.201) on Shannon Diversity Index. Finally, the quality of water is classified according to this index in the middle level.
\end{abstract}

Keywords Phytoplankton diversity $\cdot$ Abundance $\cdot$ Seasonality $\cdot$ The Caspian Sea

\section{Introduction}

The Caspian Sea is regarded as the largest close lake with brakishwater on Earth (Grigorovich et al. 2003). Ecological and morphological traits of the open seas are not seen in this ecosystem; this is regarded as a sea because of its vast area. Based on physical, geographical, and hydrological characteristics, the Caspian Sea is classified into three parts: Northern, Central and Southern Caspian. Rivers like Sefidrud, Gorganrud, Tajan, Haraz, Shiroud, Sardabrud, Talar, Babolrud, etc. are discharged into the Caspian Sea. One of the unique features of the Caspian Sea habitat is the diversity of its origins. The most abundant species are endemic (about $75 \%$ of the total number) and a few from Mediterranean (6\%) and the Arctic (3\%), and also the immigrants from freshwater. About 15 phytoplankton species have been introduced to the Caspian Sea, as the

N. Heydari · S. M. R. Fatemi $(\bowtie) \cdot$ A. Mashinchian

Department of Natural Resources and Environment, Science and Research Branch, Islamic Azad University, Tehran, Iran

e-mail: reza_fatemi@hotmail.com

R. M. Nadushan

Department of Fishery, Marine Science and Technology Campus, Islamic Azad University, North Branch of Tehran,

Zaaferaniyeh, Tehran, Iran

B. Raeisi

Department of Mathematics, Basic Sciences School, Shahed University, Tehran, Iran 
result of the artificial connecting of the Caspian Sea with the Azov-Black Sea basin by the Volga-Don Canal since 1952 (Kosarev and Yablonskaya 1994).

Bandar-e Anzali is the largest port in northern Iran and is one of the most active ports of the Caspian Sea. Anzali's main importance is its harbor location, which plays an important role in the transportation of water of the Caspian Sea, fisheries and aquatic environments on the southern shores of the Caspian Sea (Dumont 1998). Phytoplankton is considered as an important and main element in aquatic environments, since it composes the first ring of the food chain in the ecosystem (Boni 1925). Phytoplanktons are useful bioindicators of changing environmental conditions and the advantage of using biological indicators over chemical or physical ones (Griffith et al. 2002). On the other hand, a number of introduced species have ecological negative effects on ecosystems. Therefore, the identification and qualitative studies of phytoplankton populations not only have an important role in a collection of historical data, but are also necessary to understand the polluted and under stress environment of the Caspian Sea (Nasrollahzadeh Saravi et al. 2014).

Phytoplankton is beneficial to evaluate the lakes because of suitable characters such as short life cycle, high cell abundance and species diversity, sensitivity to different kinds of organic and inorganic pollution, various zone of tolerance against contaminates and environmental stressors (Castro and Huber 1991).

Primary production in the Caspian Sea is dependent on nutrients content and type of compounds in sea water. Most nutrient compounds are entered through rivers and drainages, as annual discharge of silica, phosphorous and nitrogenous into the Caspian Sea by the rivers is 95,90 , and $80 \%$, respectively. Vertical distribution of the nutrients in deep waters of the Central and Southern Caspian Sea is influenced by the intense of water mixing resulted from convection flow and increased with increased depth (Ghasemov and Bagherov 1983). In the Caspian Sea, 449 phytoplankton species consist of 163 diatoms, 139 Chlorophytes, 102 Cyanophytes, 39 Dinofagellates, 5 Euglenophytes and 1 Chrysophyta during 1962-1974. In addition, different kinds of algae have been identified as 414 species in the north, 225 species in the center and 71 species in the south.

Therefore, the number of phytoplankton is decreased from north to south due to a decline in the number of freshwater phytoplankton species (Proshkina-Lavrenko and Makarova 1968; Kosarev and Yablonskaya 1994). Of the Caspian phytoplankton compounds, diatoms are dominant in species number (163 species), but their population becomes less than green algae at the northern Caspian in summer for some years (Ghasemov and Bagherov 1983; Salmanov 1987).

Phytoplankton distribution in this ecosystem is not uniform during different seasons of the year (Salmanov 1987). At present, there are several problems in this sensitive and vulnerable ecosystem which are considered as major environmental concerns.

Population growth of cities in the Caspian basin, increased discharge of different wastewaters (industrial, agricultural and civil effluents), oil extraction development and the connection of this sea with the open seas through Volga-den canal have caused many problems (Bagheri et al. 2010) like as increased water temperature, comb jellyfish invasion, and eutrophication at the end of twentieth century (Izaguirre et al. 2001). Phytoplankton is the main group that plays a dynamic and active role in the marine food web, in exploiting primary products at higher levels of nutrients and elements in the global cycle of crops. By itself, it contains $1 \%$ of organic carbon and $45 \%$ of annual primary production on Earth. Phytoplankton include proteins, carbohydrates, lipids, vitamins and mineral salts, that they are directly and indirectly considered as food sources for all marine organisms. In addition, the oxygen produced by the phytoplankton is a result of photosynthesis, a vital component of the system on the Earth (Falkowski and Raven 1997).

Hence, the goal of this investigation was to identify species diversity and abundance of the main phytoplankton groups in south west of the Caspian. Finally, this present study shows data of phytoplankton in recent years.

\section{Materials and methods}

The present survey was conducted in Bandar-e Anzali in the west southern Caspian Sea $\left(37^{\circ} 0.27^{\prime} 59^{\prime \prime} \mathrm{N}\right.$ $49^{\circ} 0.27^{\prime} 59^{\prime \prime} \mathrm{E}$ ) and has a coastline of $40 \mathrm{~km}$. Sampling was performed at 12 stations located at four transects (Hasanrod, Taleb abad, Bandar coast, Sangachin) (a half line perpendicular to the coast) with 3 stations at 


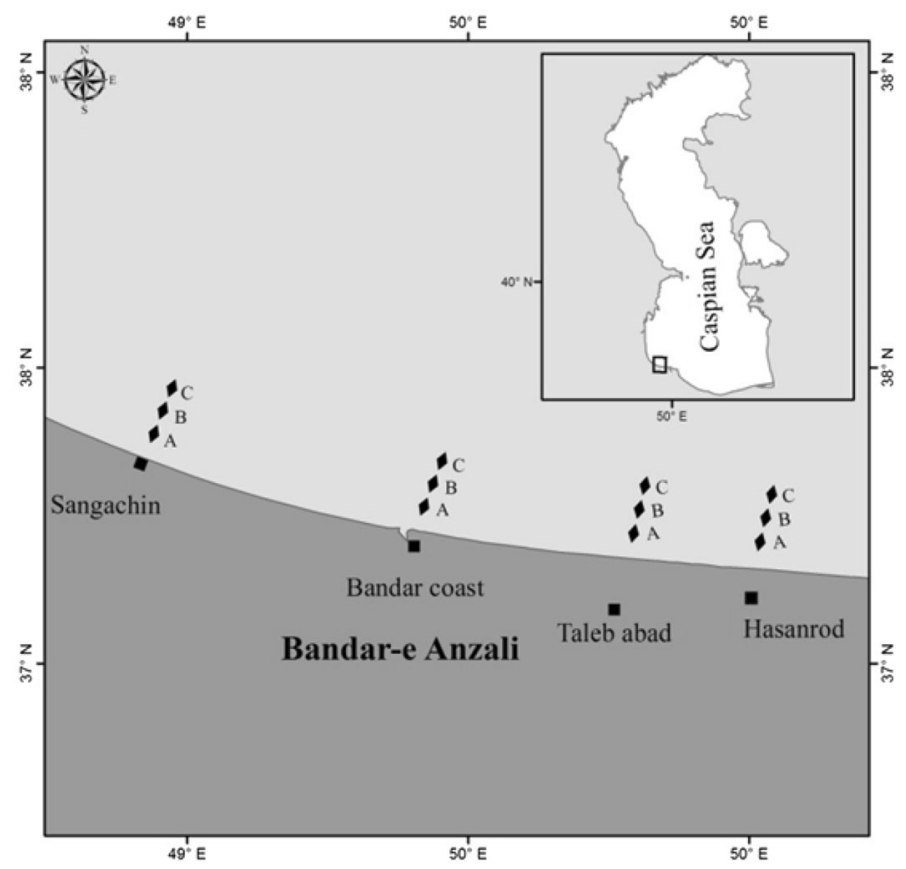

Fig. 1 Bandar-e Anzali Map from the southwestern Caspian Sea, showing the sampling sites

Table 1 Sampling transects and the location of stations in Bandar-e Anzali from the southwestern Caspian Sea

\begin{tabular}{llllr}
\hline Transect & Station & Depth $(\mathrm{m})$ & Longitude & Latitude \\
\hline Hasan rod & A & Surface water $(0)$ & 49.40 .55 & 37.27 .21 \\
& B & 5 & 49.40 .49 & 37.27 .35 \\
Talebabad & C & 10 & 49.40 .36 & 37.27 .51 \\
& A & Surface water (0) & 49.32 .53 & 37.28 .04 \\
Anzali coast & B & 5 & 49.32 .57 & 37.28 .16 \\
& C & 10 & 49.33 .01 & 37.28 .39 \\
Sangachin & A & Surface water (0) & 49.27 .20 & 27.28 .53 \\
& B & 5 & 49.27 .25 & 37.29 .10 \\
& C & 10 & 49.27 .26 & 37.29 .20 \\
& A & Surface water (0) & 37.31 .06 \\
& B & 5 & 49.19 .04 & 37.31 .22 \\
& C & 10 & 49.19 .19 & 37.31 .40 \\
\hline
\end{tabular}

surface water, depths of 5 and $10 \mathrm{~m}$ (Fig. 1, Table 1). The sampling stations were fixed near shore. Sampling was performed seasonally, during spring 2012 to spring 2013.

In order to study phytoplankton, water samples were collected in Van Dorn water bottle (Ruttner) (Vollenweider 1974) and poured into plastic containers. Then, $7 \mathrm{ml}$ of Lugol iodine solution was added to the containers $(0.7 \mathrm{ml}$ per each $100 \mathrm{ml}$ of the sample) and after $1 \mathrm{~h}, 40 \mathrm{ml}$ of $40 \%$ concentrated formaldehydes was added to the vessels. Therefore, the final solution contained $4 \%$ of formalin (APHA 2005). Three replicates were obtained at each station from surface water, with 5 and $10 \mathrm{~m}$ depth.

Phytoplankton samples were transported to the laboratory and stayed for $48 \mathrm{~h}$. Then, the supernatant was siphoned in order to make the sample denser. The remaining was centrifuged for several times for 5 min at $3000 \mathrm{rpm}$ to gain $30 \mathrm{ml}$ volume of each sample (Centrifuge Model Number D-7200 tutilinge7).

To identify phytoplankton species, each sample was shaken well to obtain a homogenized sample. Thereafter, three replicates of each sample $(1 \mathrm{ml})$ were pipetted from the $30 \mathrm{ml}$ sample and poured into a Sedgewick-Rafter counting chamber under an invert microscope (Vollenweider 1974; Newell 1977). Species 
identification was performed using valid identification keys (Prescott 1962; Kasymov 2000; Tiffany and Britton 1971). Statistical analysis was done using the SPSS software (version 11.5). The data were tested to check for the normality. One-way analysis of variance (ANOVA) was used for the existence of significant differences $(P<0.05)$ between transects seasons and depths. Multivariate regression was used in this study. Phytoplankton phyla were considered as dependent variables and transect and depth were considered as independent variables.

Discriminate function analysis was applied to indicate how different abundances of phytoplankton phyla vary across transect. Shannon-Wiener Index was used to study species diversity. The values of this index are divided into five qualitative classes (Marques et al. 2009) (Table 4). Shanon Index is calculated by the following formula:

$$
H^{\prime}=-\Sigma(n i / N) \ln (n i / N),
$$

$N$ is the total population density, ni is the species density $i$.

\section{Results}

In this trail, 72 species from 59 genera, 45 families, 31 orders, and 5 main phytoplankton branches were identified (Table 2). Of these identified phytoplankton, 7 orders, 11 families, 16 genera, and 17 species were allocated to Green algae (Chlorophyta); 3 orders, 5 families, 10 genera and 6 species to blue-green algae (Cyanophyta); 5 orders, 6 families, 7 genera and 16 species to Dinoflagellata (Pyrrophyta); 1 order, 1 family, 3 genera, and 4 species to Euglenoids (Euglenophyta); 15 orders, 17 families, 23 genera and 29 species to diatoms (Bacillariophyta).

Among the phytoplankton species, 29 species (40\%) were from diatoms (Bacillariophyta), 17 species (24\%) from green algae (Chlorophyta), 16 species (22\%) from Dinoflagellata (Pyrrophyta), 6 species (8\%) from blue-green algae (Cyanophyta) and 4 species (6\%) from Euglenophyta. The number of recorded species in spring, summer, autumn, and winter was $46,50,41$ and 39 , respectively.

Diatoms showed the most species diversity in winter (22 species) and autumn (21 species), while the lowest one was observed in spring (13 species). The highest species number of Pyrrophyta was detected in spring (15 species), Cyanobacteria in summer (6 species), Chlorophyta in spring (14 species) and Euglenophyta in summer (4 species) (Table 3).

Seasonally phytoplankton diversity in the southern Caspian Sea in Bandar-e Anzali was investigated based on Shannon-Diversity Index in the present study and exhibited the most diversity index in Autumn (2.532) and the least index in Spring (2.201) with an annual mean of this index (2.8) (Fig. 2). Eventually, Shannon index in Bandar-e Anzali is classified according to Table 4 in the moderate class.

During the first three seasons of the year, the highest abundance was discovered at $10 \mathrm{~m}$ depth while in winter it was observed at depths of 5 and $10 \mathrm{~m}$. In different depths the most and the lowest abundance were recorded at depth of 10 and surface water, respectively. Transect and depth had significant effect on density of phytoplankton phyla $(P<0.05)$ (Table 5), but the interaction effect of depth by transect showed no significant effect $(P>0.05)$ (Fig. 3). The number of Cyanobacteria and Euglenophyta species is completely similar in different transects located at the southern Caspian Sea, whereas the species number of diatoms was variable at different transects. 12 species of diatoms, 9 species of Pyrrophyta and 13 species of other branches were revealed in Sangachin (Fig. 4). In fact, the main phytoplankton groups with most species number in Bandar-e Anzali were diatoms, green algae and Dinoflagellata. Moreover, Sangachin and Bandar coast transects had the highest and the least species diversity and cell abundance, respectively.

Discriminant Function Analysis was applied to better find any differences among transects based on phytoplankton cell abundance. As depicted in Fig. 5, DFA could indicate the discrimination between transect 1 (Hasan rod) and 3 (Bandar coast) as well as transect 2(Taleb abad) and 4(Sangachin). Transect 3 was well discriminated from other transects.

According to the results of DFA, among phytoplankton phyla isolated from the Caspian Sea, three phyla including Cyanophyta, Pyrrophyta and Euglenophyta had major role in discriminating the transects as their abundance among various transects showed significant differences $(P<0.05)$. 
Table 2 Identified phytoplankton species from southern Caspian Sea in all seasons

\begin{tabular}{|c|c|c|c|c|}
\hline Phylum & Order & Family & Genus & Species \\
\hline \multirow[t]{18}{*}{ Chlorophyta } & Chlorellales & Oocystaceae & (1) Gloeotaenium & (1) loitlesbergerianum \\
\hline & \multirow[t]{4}{*}{ Chlorellales } & \multirow[t]{4}{*}{ Oocystaceae } & \multirow[t]{3}{*}{ (2) Oocystis } & (2) borgi \\
\hline & & & & $\mathrm{sp}$ \\
\hline & & & & (3) solitaria \\
\hline & & & (3) Codotella & $\mathrm{sp}$ \\
\hline & Sphaeropleales & Hydrodictyaceae & (4) Pediastrum & (4) tetras \\
\hline & Chlorellales & Botryococcaceae & (5) Botryococcus & $\mathrm{sp}$ \\
\hline & \multirow[t]{2}{*}{ Sphaeropleales } & \multirow[t]{2}{*}{ Scenedesmaceae } & \multirow[t]{2}{*}{ (6) Scenedesmus } & (5) quadricauda \\
\hline & & & & (6) abundans \\
\hline & Zygnematales & Zygnemataceae & (7) Spirogira & $\mathrm{sp}$ \\
\hline & \multirow[t]{2}{*}{ Sphaeropleales } & \multirow[t]{2}{*}{ Selenastraceae } & \multirow[t]{2}{*}{ (8) Ankistrodesmus } & (7) arcuatus \\
\hline & & & & $\mathrm{sp}$ \\
\hline & Desmidiales & Closteriaceae & (9) Closterium & (8) moniliferum \\
\hline & \multirow[t]{5}{*}{ Chlamydomonadales } & \multirow[t]{5}{*}{ Chlamydomonadacea } & \multirow[t]{5}{*}{ (10) Chlamydomonas } & (9) globosa \\
\hline & & & & $\mathrm{sp}$ \\
\hline & & & & (10) flusa \\
\hline & & & & (11) ovalis \\
\hline & & & & (12) monasovalis \\
\hline \multirow[t]{7}{*}{ Chlorophyta } & Chlorococcales & Chlorococcaceae & (11) Selenstrum & (13) bibraianum \\
\hline & Ulotrichales & Ulotrichaceae & (12) Binuclearia & (14) lauterbomii \\
\hline & Sphaeropleales & Scenedesmaceae & (13) Coelastrum & (15) sphaericum \\
\hline & Chlorococcales & Chlorococcaceae & (14) Scheroderia & (16) setigera \\
\hline & Chlorellales & Chlorellaceae & (15) Chlorella & $\mathrm{sp}$ \\
\hline & \multirow[t]{2}{*}{ Chlorococcales } & \multirow[t]{2}{*}{ Scenedesmaceae } & \multirow[t]{2}{*}{ (16) Crucigenia } & $\mathrm{sp}$ \\
\hline & & & & (17) quadrata \\
\hline \multirow[t]{16}{*}{ Cyanophyta } & \multirow[t]{2}{*}{ Chlorococcales } & \multirow[t]{2}{*}{ Microcystaceae } & \multirow[t]{2}{*}{ (17) Microcystis } & (18) -1aeruginosa \\
\hline & & & & $\mathrm{sp}$ \\
\hline & \multirow[t]{2}{*}{ Oscillatoriales } & Oscillatoriaceae & (18) Oscillatoria & (19) -2limosa \\
\hline & & & & $\mathrm{sp}$ \\
\hline & Nostocales & Oscillatoriaceae & (19) Lyngbya & (20) -3limnetica \\
\hline & & & & $\mathrm{sp}$ \\
\hline & Chlorococcales & Chlorococcaceae & (20) Aphanothece & $\mathrm{sp}$ \\
\hline & Nostocales & Nostocaceae & (21) Nodularia & $\mathrm{sp}$ \\
\hline & Nostocales & Nostocaceae & (22) Anabeanopsis & (21) -4arnoldii \\
\hline & & & & $\mathrm{sp}$ \\
\hline & & & & (22) -5rasiburaskii \\
\hline & Nostocales & Nostocaceae & (23) Anabaena & sp \\
\hline & & & & (23) -6reniformis \\
\hline & Chlorococcales & Merismopediaceae & (24) Merismopedia & $\mathrm{sp}$ \\
\hline & Chlorococcales & Microcystaceae & (25) Gloeocapsa & $\mathrm{sp}$ \\
\hline & Nostocales & Oscillatoriaceae & (26) Spirulina & $\mathrm{sp}$ \\
\hline Pyrrophyta & Peridiniales & Glenodiniaceae & (27) Glenodinium & (24) -1 cinctum \\
\hline & & & & (25) -2behningii \\
\hline & & & & $\mathrm{sp}$ \\
\hline & & & & (26) -3penardii \\
\hline & Peridiniales & Peridiniaceae & (28) Peridinium & (27) -4subsalum \\
\hline & & & & (28) -5trochoideum \\
\hline & & & & $\mathrm{sp}$ \\
\hline
\end{tabular}


Table 2 continued

\begin{tabular}{|c|c|c|c|c|}
\hline Phylum & Order & Family & Genus & Species \\
\hline & & & & (29) -6achromaticum \\
\hline & Prorocentrales & Prorocentraceae & (29) Exuviaella & (30) -7 cordata \\
\hline & Prorocentrales & Prorocentraceae & (30) Prorocentrum & (31) -8paraximum \\
\hline & & & & (32) -9cordatum \\
\hline & & & & (33) -10scutelum \\
\hline & & & & (34) -11micans \\
\hline & Gonyaulacales & Gonyaulacaceae & (31) Goniaulax & (35)-12digitale \\
\hline & & & & $\mathrm{sp}$ \\
\hline & & & & (36) -13polyedra \\
\hline & & & & (37) -14spinifera \\
\hline & Gymnodiniales & Gymnodiniacea & (32) Gymnodinium & (38) -15variabile \\
\hline & Chattonellales & Vacuolariaceae & (33) Gonyastomum & (39) -16depressum \\
\hline \multirow[t]{7}{*}{ Euglenophyta } & Euglenales & Euglenaceae & (34) Euglena & $\mathrm{sp}$ \\
\hline & & & & (40) -1wangi \\
\hline & & & & (41) $-2 t u b a$ \\
\hline & Euglenales & Euglenaceae & (35) Phacus & $\mathrm{sp}$ \\
\hline & Euglenales & Euglenaceae & (36) Trachelomonas & $\mathrm{sp}$ \\
\hline & & & & (42) -3planctoniea \\
\hline & & & & (43) -4slimilis \\
\hline \multirow[t]{13}{*}{ Diatoms } & Thalassiosirales & Thalassiosiraceae & (37) Thalassiosira & (44) -1hustdti \\
\hline & & & & $\mathrm{sp}$ \\
\hline & Thalassiosirales & Skeletonemaceae & (38) Rhizosolenia & (45) -2fragilissima \\
\hline & & & & (46) -3calcaravis \\
\hline & Thalassionematales & Thalassionemataceae & (39) Thalassionema & (47) -4nitzschiodes \\
\hline & Bacillariales & Bacillariaceae & (40) Nitzschia & (48) -5 seriata \\
\hline & & & & (49) -6reversa \\
\hline & & & & (50)-7acicularis \\
\hline & & & & $\mathrm{sp}$ \\
\hline & Bacillariales & Bacillariaceae & (40) Nitzschia & (51) -8tenirustris \\
\hline & & & & (52) -9sigmoidea \\
\hline & Thalassiosirales & Skeletonemaceae & (41) Skletonema & (53) -10subsalum \\
\hline & Thalassiosirales & Skeletonemaceae & & (54) -11costatum \\
\hline \multirow[t]{14}{*}{ Diatoms } & Naviculales & Naviculaceae & (42) Navicula & $\mathrm{sp}$ \\
\hline & Fragilariales & Fragilariaceae & (43) Synedra & (55) $-12 u \ln a$ \\
\hline & Centrales & Chaetocerotaceae & (44) Chaetoceros & $\begin{array}{l}\text { (56)-13muelleri } \\
\mathrm{sp}\end{array}$ \\
\hline & Tribonematales & Tribonemataceae & (45) Tribonema & (57) -14vulgar \\
\hline & Thalassiosirales & Stephanodiscaceae & (46) Cyclotella & (58) -15menenghiniana \\
\hline & Cymbellales & Cymbellaceae & (47) Cymbella & (59) -16tumidae \\
\hline & & & & $\mathrm{sp}$ \\
\hline & Coscinodiscales & Coscinodiscaceae & (48) Coscinodiscus & (60) -17perforatus \\
\hline & & & & (61) -18gigas \\
\hline & Thalassiophysales & Catenulaceae & (49) Amphora & $\mathrm{sp}$ \\
\hline & Naviculales & Diploneidaceae & (50) Diplonois & (62) -19interupta \\
\hline & ntholes & Cocconeidacene & (51) Соссопоіс & sp \\
\hline & Renanantuates & Carcunierilatede & (Ji) cocconets & $\mathrm{sp}$ \\
\hline & & & & (64) -21placentula \\
\hline
\end{tabular}


Table 2 continued

\begin{tabular}{|c|c|c|c|c|}
\hline \multirow[t]{2}{*}{ Phylum } & \multirow[t]{2}{*}{ Order } & \multirow[t]{2}{*}{ Family } & \multirow[t]{2}{*}{ Genus } & \multirow{2}{*}{$\frac{\text { Species }}{(65)-22 \text { husteli }}$} \\
\hline & & & & \\
\hline & Pennales & Fragilariaceae & (52) Diatoma & $\mathrm{sp}$ \\
\hline & Melosirales & Melosiraceae & (53) Melosira & (66) -23moniliformis \\
\hline & & & & (67) -24juergensii \\
\hline & & & & (68) -25granulata \\
\hline & & & & (69) -26varians \\
\hline & Fragilariales & Fragilariaceae & (54) Fragilaria & (70) -27capucina \\
\hline & Chromulinales & Dinobryaceae & (55) Dinobryon & $\mathrm{sp}$ \\
\hline & Naviculales & Pleurosigmataceae & (56) Gyrosigma & (71) -28attenuatum \\
\hline & Naviculales & Pleurosigmataceae & (56) Pleurosigma & $\mathrm{sp}$ \\
\hline & & & & (72) -29elongatum \\
\hline & Thalassiosirales & Stephonodiscacaeae & (58) Stephonodiscus & $\mathrm{sp}$ \\
\hline & Surirellales & Surirellaceae & (59) Surirella & $\mathrm{sp}$ \\
\hline
\end{tabular}

The following equations obtained from DFA indicate the relationship between phytoplankton phyla and transects.

$$
\begin{aligned}
\text { DA1 }= & -0.252 \text { chlorophyta }+0.495 \text { cyanophyta } \\
& +0.846 \text { pyrrophyta }+0.295 \text { euglenophyta }+0.363 \text { diatoms } \\
\text { DA2 }= & 0.755 \text { chlorophyta }+0.168 \text { cyanophyta } \\
& +0.176 \text { pyrrophyta }-1.279 \text { euglenophyta }+0.280 \text { diatoms } \\
\text { DA3 }= & 0.082 \text { chlorophyta }+0.998 \text { cyanophyta } \\
& -0.461 \text { pyrrophyta }-0.392 \text { euglenophyta }+0.322 \text { diatoms }
\end{aligned}
$$

The following equations obtained from DFA indicate the relationship between phytoplankton phyla and depths.

$$
\begin{aligned}
\text { DA1 }= & 1.092 \text { chlorophyta }+0.203 \text { cyanophyta } \\
& +0.157 \text { pyrrophyta }-0.570 \text { euglenophyta }+0.459 \text { diatoms } \\
\text { DA2 }= & -0.871 \text { chlorophyta }+0.817 \text { cyanophyta } \\
& +0.502 \text { pyrrophyta }+0.604 \text { euglenophyta }+0.238 \text { diatoms. }
\end{aligned}
$$

The overall total average of cell abundance was approximately $43.55 \pm 5.10 \times 10^{4}$ cells $/ \mathrm{m}^{3}$. Actually, annual average dominance of cell abundance was discovered in diatoms with $14.99 \pm 16.97 \times 10^{4}$ cells $/ \mathrm{m}^{3}$ (Fig. 6). Because of high abundance of diatoms, the highest average abundance was detected in winter related to Thalassionema nitzschiodes at a depth of $10 \mathrm{~m}$. The lowest and the highest cell abundance of diatoms were reported in summer and winter $\left(5970\right.$ cells $/ \mathrm{m}^{3}$ and 359,370 cells $/ \mathrm{m}^{3}$, respectively) (Fig. 7) and their cell abundance showed significant differences at various seasons $(P<0.05)$.

Of Dinoflagellata, the most and least cell abundance was in spring $\left(144,720\right.$ cells $\left./ \mathrm{m}^{3}\right)$ and autumn $(44,790$ cells $\left./ \mathrm{m}^{3}\right)$ with significant differences among seasons $(P<0.05)$ and the highest cell abundance was of Exuviaella cordata at a depth of $10 \mathrm{~m}$ (Fig. 7). By contrast, Cyanobacteria had their highest cell abundance in spring $\left(25,792.5\right.$ cells $\left./ \mathrm{m}^{3}\right)$ and their lowest was displayed in summer $\left(187,890 \mathrm{cells} / \mathrm{m}^{3}\right)$. and highest cell abundance related to Oscillatoria limosa at a depth of $10 \mathrm{~m}$. There were significant differences in cell abundance of this group among various seasons $(P<0.05)$ (Fig. 7). In spring and summer, the lowest $(14,490$ cells $\left./ \mathrm{m}^{3}\right)$ and the most cell abundance $\left(107,610 \mathrm{cells} / \mathrm{m}^{3}\right)$ of green algae were observed, respectively. And this index exhibited a significant difference between summer with other seasons $(P<0.05)$ whereas no significant 
Table 3 Number of identified phytoplankton species from southern Caspian Sea in different seasons

Seasons

Spring
Summer

Chlorophyta

1. Ankistrodesmus arcuatus

2. Ankistrodesmus sp.

3. Binuclearia lauterbomii

4. Botryococcus sp.

5. Chlamydomonas globosa

6. Chlamydomonas flusa

7. Chlamydomonas monasovalis

8. Chlamydomonas ovalis

9. Chlamydomonas sp.

10. Chlorella sp.

11. Closterium moniliferum

12. Codotella sp.

13. Coelastrum sphaericum

14. Crucigenia quadrata

15. Crucigenia $\mathrm{sp.}$

16. Gloeotaenium loitlesbergerianum

17. Oocystis borgi

18. Oocystis solitaria

19. Oocystis sp.

20. Pediastrum tetras

21. Scenedesmus abundans

22. Scenedesmus quadricauda

23. Scheroderia setigera

24. Selenstrum bibrajanum

Total

Cyanophyta

1. Anabaena reniformis

2. Anabaena sp.

3. Anabaenopsis arnoldii

4. Anabeanopsis raciborskii

5. Anabeanopsis sp.

6. Aphanothece sp.

7. Gloeocapsa sp.

8. Lyngbya limneticula

9. Lyngbya sp.

10. Merismopedia sp.

11. Microcystis aeruginosa

12. Microcystis sp.

13. Nodularia sp.

14. Oscillatoria limosum

15. Oscillatoria sp.

16. Spirulina sp.

Total

Pyrrophyta

1. Exuviaella cordata

2. Glenodinium behningii
$+$

$+$

$+$

$+$

$+$

$+$

$+$

$+$

$+$

$+$

$+$

$+$

$+$

$+$

$+$

$+$

$-$

$+$

$-$

$+$

$+$

$-$

$+$

20

$\begin{array}{ll}- & + \\ - & +\end{array}$

$+$

$-$

$-$

$+$

$+$

$+$

$+$

$+$

$+$

$-$

$+$

$+$

$+$

$+$

$+$

12

15

Autumn

Winter

(

$-$

$-$

$+$

$-$

$-$

$+$

$-$

-

$+$

$+$

$+$

$-$

$+$

$-$

$-$

$-$

$-$

$-$

$+$

$-$

$-$

$-$

$+$

$+$

$+$

9

$-$

$+$

$+$

$+$

$-$

$-$

$-$

$+$

$+$

$+$

$+$

$+$

$+$

$-$

$-$

-

$+$

$-$

$+$

$+$

$-$

-

$-$

$-$

12

$+$

$+$

$-$

$+$

$+$

$-$

$-$

$+$

$+$

$+$

$+$

-

$-$

$+$

+
+

+
+

+
11

12

1

\begin{tabular}{llll}
+ & + & + & + \\
\hline
\end{tabular}


Table 3 continued

\begin{tabular}{|c|c|c|c|c|}
\hline & \multicolumn{4}{|c|}{ Seasons } \\
\hline & Spring & Summer & Autumn & Winter \\
\hline 3. Glenodinium cinctum & + & - & - & - \\
\hline 4. Glenodinium penardii & + & - & - & - \\
\hline 5. Glenodinium sp. & + & - & + & + \\
\hline 6. Goniaulax digitale & + & - & - & + \\
\hline 7. Goniaulax polyedra & + & - & + & - \\
\hline 8. Goniaulax spinifera & - & - & + & - \\
\hline 9. Goniaulax sp. & + & - & + & + \\
\hline 10. Gonyastomum depressum & + & + & - & - \\
\hline 11. Gymnodinium variabile & + & - & + & + \\
\hline 12. Peridinium achromaticum & + & + & + & + \\
\hline 13. Peridinium subsalum & + & - & - & - \\
\hline 14. Peridinium trochoideum & + & - & - & - \\
\hline 15. Peridinium sp. & + & - & + & + \\
\hline 16. Prorocentrum cordatum & + & + & - & - \\
\hline 17. Prorocentrum micans & + & + & + & + \\
\hline 18. Prorocentrum paraximum & + & + & + & + \\
\hline 19. Prorocentrum scutellum & + & + & + & + \\
\hline Total & 17 & 7 & 11 & 10 \\
\hline \multicolumn{5}{|l|}{ Euglenophyta } \\
\hline 1. Euglena tuba & + & + & + & - \\
\hline 2. Euglena wangi & + & + & - & - \\
\hline 3. Euglena sp. & + & + & + & + \\
\hline 4. Phacus sp. & + & + & + & + \\
\hline 5. Trachelomonas planctoniea & - & + & - & - \\
\hline 6. Trachelomonas slimilis & - & + & - & + \\
\hline 7. Trachelomonas sp. & + & + & + & + \\
\hline Total & 5 & 7 & 4 & 4 \\
\hline \multicolumn{5}{|l|}{ Bacillariophyta } \\
\hline 1. Amphora sp. & - & - & - & + \\
\hline 2. Chaetoceros muelleri & + & - & + & + \\
\hline 3. Chaetoceros sp. & - & - & + & + \\
\hline 4. Cocconeis husteli & - & + & - & - \\
\hline 5. Cocconeis placentula & - & + & - & + \\
\hline 6. Cocconeis scutellum & - & + & - & - \\
\hline 7. Cocconeis sp. & - & - & + & + \\
\hline 8. Coscinodiscus gigas & - & + & + & + \\
\hline 9. Coscinodiscus perforatus & + & - & + & + \\
\hline 10. Cyclotella meneghiniana & + & + & + & + \\
\hline 11. Cymbella tumidae & - & + & + & + \\
\hline 12. Cymbella sp. & + & - & + & + \\
\hline 13. Diatoma sp. & + & - & - & - \\
\hline 14. Dinobryon sp. & + & - & - & - \\
\hline 15. Diplonois interupta & - & + & + & + \\
\hline 16. Diplonois sp. & - & + & + & - \\
\hline 17. Fragilaria capucina & - & + & - & - \\
\hline 18. Gyrosigma attenuatum & + & - & + & + \\
\hline 19. Melosira granulate & - & + & + & - \\
\hline
\end{tabular}


Table 3 continued

\begin{tabular}{|c|c|c|c|c|}
\hline & \multicolumn{4}{|c|}{ Seasons } \\
\hline & Spring & Summer & Autumn & Winter \\
\hline 20. Melosira juergensii & - & + & - & - \\
\hline 21. Melosira moniliformis & - & + & - & - \\
\hline 22. Melosira varians & - & - & + & + \\
\hline 23. Navicula sp. & + & + & + & + \\
\hline 24. Nitzschia acicularis & + & + & + & + \\
\hline 25. Nitzschia reversa & - & + & - & + \\
\hline 26. Nitzschia seriata & + & - & + & + \\
\hline 27. Nitzschia sigmoidea & + & + & + & + \\
\hline 28. Nitzschia tenirustris & - & + & - & + \\
\hline 29. Nitzschia sp. & + & - & + & + \\
\hline 30. Pleurosigma elongatum & - & + & + & - \\
\hline 31. Pleurosigma sp. & - & - & + & + \\
\hline 32. Rhizosolenia fragilissima & + & - & + & + \\
\hline 33. Rhizosolenia calcaravis & + & - & + & + \\
\hline 34. Skeletonema costatum & - & + & + & + \\
\hline 35. Skeletonema subsalum & - & + & + & + \\
\hline 36. Stephonodiscus sp. & - & - & + & - \\
\hline 37. Surirella sp. & - & - & + & + \\
\hline 38. Synedra ulna & + & - & + & + \\
\hline 39. Thalassionema nitzschiodes & + & - & + & + \\
\hline 40. Thalassiosira hustdti & + & - & + & + \\
\hline 41. Thalassiosira sp. & + & + & + & - \\
\hline 42. Tribonema vulgar & + & + & + & + \\
\hline Total & 19 & 22 & 31 & 30 \\
\hline Total of seasons & 73 & 69 & 67 & 67 \\
\hline
\end{tabular}

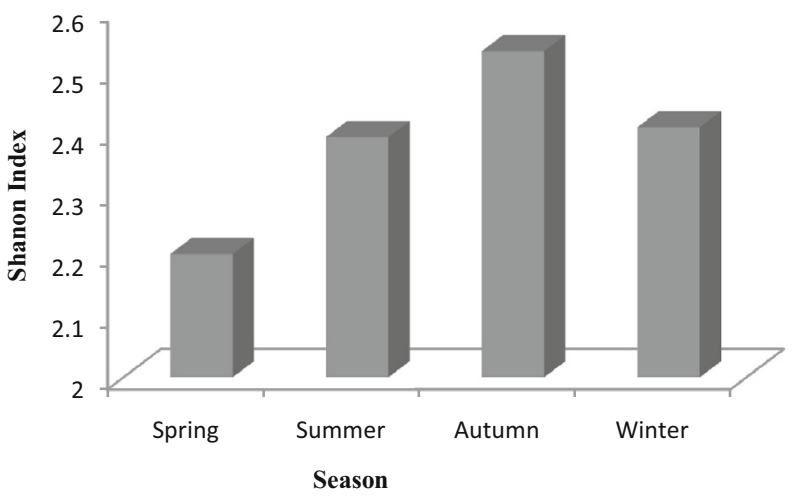

Fig. 2 Seasonality phytoplankton diversity in the southern Caspian Sea

differences were displayed among other seasons $(P>0.05)$ (Fig. 7). Euglenoids had less abundance than other phytoplankton groups and showed their lowest and the most abundance in autumn and summer (9570 cells $/ \mathrm{m}^{3}$ and 86,460 cells $/ \mathrm{m}^{3}$, respectively). Euglenoids abundance in summer revealed a significant difference 
Table 4 Water quality classification, based on Shannon Diversity Index

\begin{tabular}{lc}
\hline Rating water quality & Shannon Index \\
\hline Excellent & $>4$ \\
Good & $3-4$ \\
Moderate & $2-3$ \\
Poor & $1-2$ \\
bad & $0-1$ \\
\hline
\end{tabular}

Table 5 Multivariate tests (MANOVA) analyzing the effect of transect and depth on abundances of phytoplankton phyla in the southern Caspian Sea

\begin{tabular}{|c|c|c|c|c|c|}
\hline \multirow[t]{2}{*}{ Effect } & \multicolumn{5}{|c|}{ Multivariate tests } \\
\hline & Value & $F$ & Hypothesis $d f$ & Error $d f$ & Sig. \\
\hline \multicolumn{6}{|l|}{ Intercept } \\
\hline Pillai's trace & 0.819 & 115.691 & 5.000 & 128.000 & 0.000 \\
\hline Wilks' lambda & 0.181 & 115.691 & 5.000 & 128.000 & 0.000 \\
\hline Hotelling's trace & 4.519 & 115.691 & 5.000 & 128.000 & 0.000 \\
\hline Roy's largest root & 4.519 & 115.691 & 5.000 & 128.000 & 0.000 \\
\hline \multicolumn{6}{|l|}{ Transect } \\
\hline Pillai's trace & 0.439 & 4.457 & 15.000 & 390.000 & 0.000 \\
\hline Wilks' lambda & 0.575 & 5.231 & 15.000 & 353.753 & 0.000 \\
\hline Hotelling's trace & 0.714 & 6.026 & 15.000 & 380.000 & 0.000 \\
\hline Roy's largest root & 0.677 & 17.609 & 5.000 & 130.000 & 0.000 \\
\hline \multicolumn{6}{|l|}{ Depth } \\
\hline Pillai's trace & 0.687 & 13.508 & 10.000 & 258.000 & 0.000 \\
\hline Wilks' lambda & 0.408 & 14.496 & 10.000 & 256.000 & 0.000 \\
\hline Hotelling's trace & 1.220 & 15.496 & 10.000 & 254.000 & 0.000 \\
\hline Roy's largest root & 0.983 & 25.369 & 5.000 & 129.000 & 0.000 \\
\hline \multicolumn{6}{|l|}{ Transect $\times$ depth } \\
\hline Pillai's trace & 0.202 & 0.925 & 30.000 & 660.000 & 0.584 \\
\hline Wilks' lambda & 0.808 & 0.941 & 30.000 & 514.000 & 0.559 \\
\hline Hotelling's trace & 0.227 & 0.957 & 30.000 & 632.000 & 0.533 \\
\hline Roy's largest root & 0.167 & 3.668 & 6.000 & 132.000 & 0.002 \\
\hline
\end{tabular}

with other seasons $(P<0.05)$, while there were no significant differences among other seasons $(P>0.05)$ (Fig. 7).

At the end, the highest cell abundance was observed in the spring, summer, autumn and winter seasons that belonged to species Exuviaella cordata, Oscillatoria limosa, Thalassionema nitzschiodes and Thalassionema nitzschiodes at a depth of $10 \mathrm{~m}$, respectively.

The maximum and minimum average abundance of phytoplankton was observed in winter and spring respectively (Fig. 8).

\section{Discussion}

The main phytoplankton taxa in Caspian Sea are Bacillariophyta, Pyrrophyta and cyanophyta (Ghasemov and Bagherov 1983; Salmanov 1987; Ganjian et al. 2009; Ganjian 2007). In this study, Diatoms were the main group of phytoplankton, with the highest diversity of 29 species and $40 \%$ of the total taxa. The number of identified species was 46 in spring, 50 in summer, 41 in autumn, and 39 in winter. The highest and lowest 

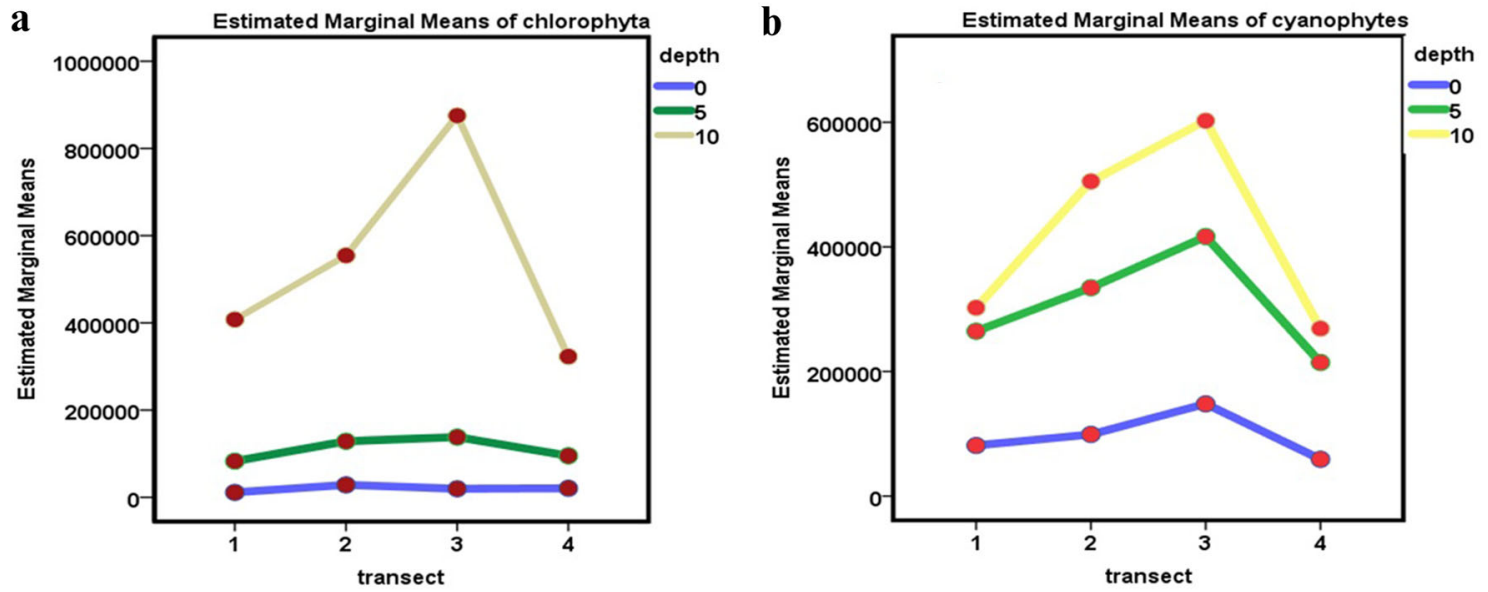

c

d
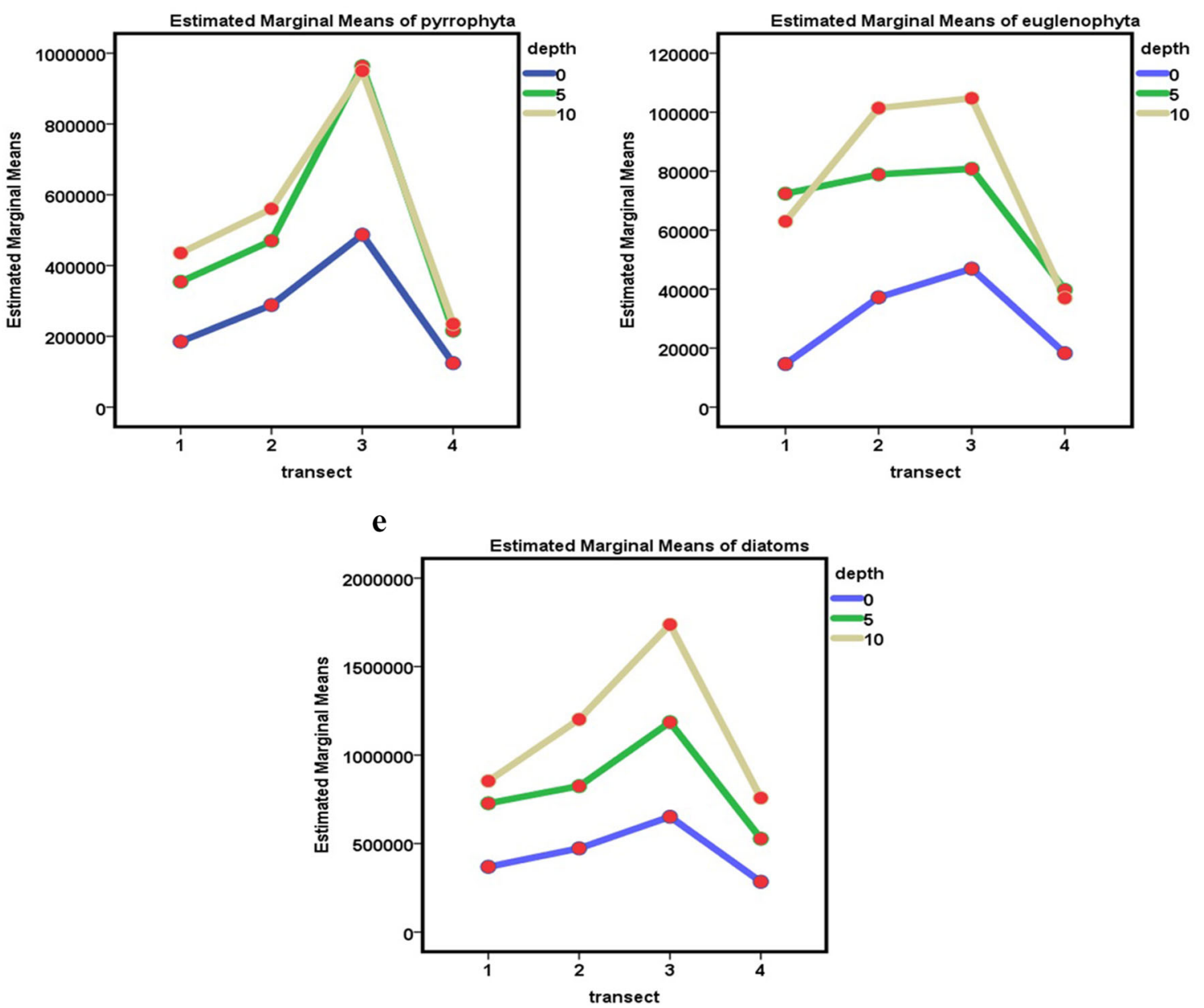

Fig. 3 Comparison between densities of phytoplankton phyla (a Chlorophyta, b Cyanophyta, c Pyrrophyta, d Euglenophyta, e Bacillariophyta) in different depths and transects

diversity was observed among phytoplankton groups in summer and autumn, respectively, which were similar to the finding in 2005-2006 recorded by Ganjian et al. 2010. They identified 101 species in the south of Caspian sea that the main phytoplankton group was Bacillariophyta (diatoms) with more than 71 species and $43 \%$ of the total taxa. The highest number of phytoplankton species was recorded in summer with 101 species in the southern part of the Caspian Sea. Temperature seems to be an important seasonal factor that controls the 


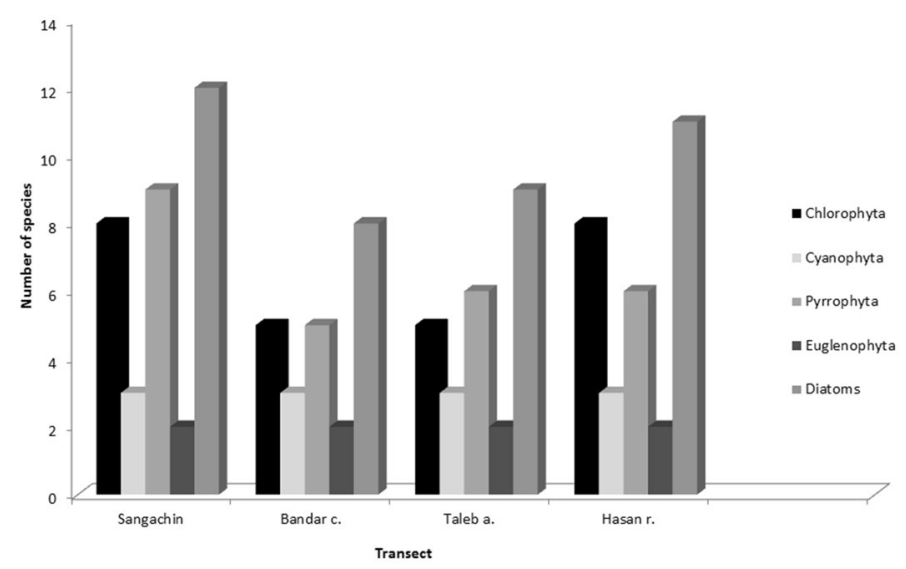

Fig. 4 Comparison between diversity of different phyla of phytoplankton in different transects

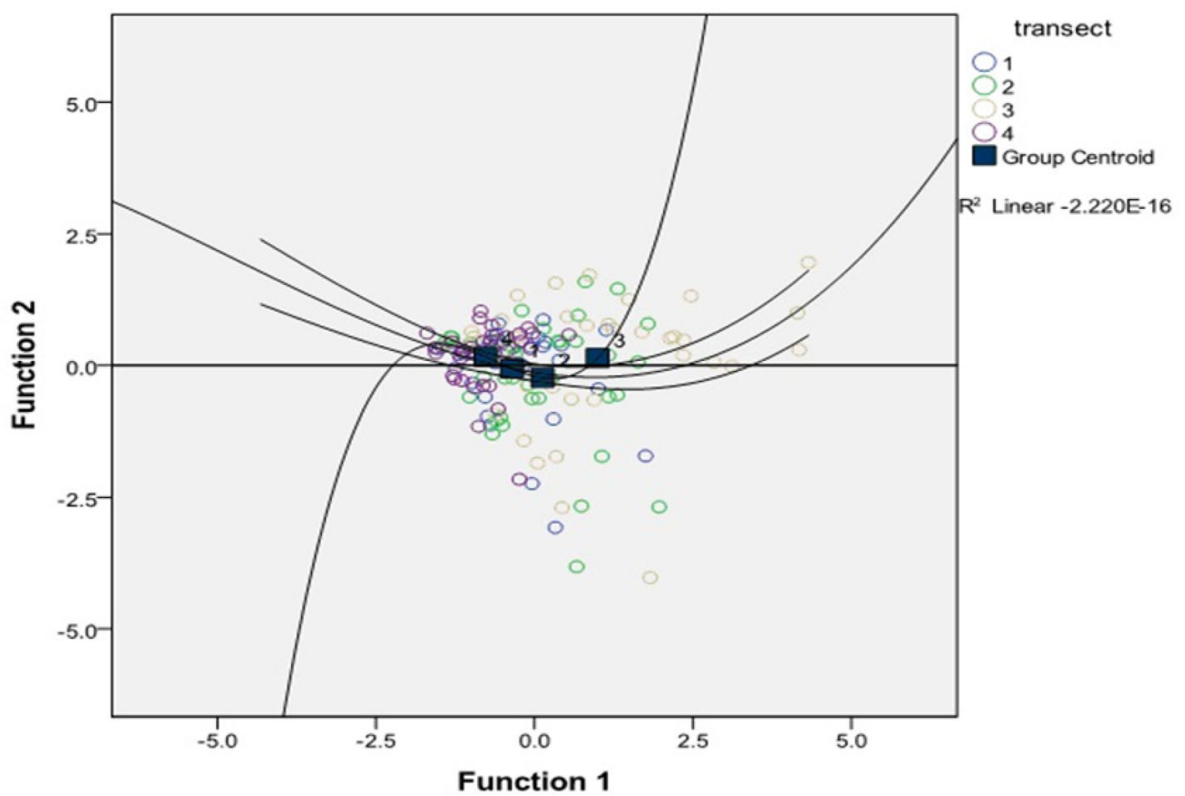

Fig. 5 Canonical Discriminant Function graph based on phytoplankton cell abundance at different transects

biological processes of phytoplankton communities (Vereshchaka and Anokhina 2014). Bat et al. (2011) reported Dinophyceae and Bacillariophyceae are the most dominant families for the number of species (respectively, 173 and 89 species) in the Black Sea. Also, Ozgur et al. (2010) published that a total of 129 taxa were recorded from neritic waters in Samsun Bay, including 76 Bacillariophyta, 2 Cyanobacteria, 1 Chlorophyta, 1 Euglenophyta and 48 other Phyla. Diatoms were dominant among phytoplankton groups except in November 2002 and June 2003.

Formerly diatoms were the most abundant in the Caspian Sea (Kosarev and Yablonskaya 1994). After the diatoms, chlorophytes and cyanophytes were the most abundant groups in the north of the Caspian Sea, while dinoflagellates dominated in the middle and southern parts of the Caspian Sea (Kosarev and Yablonskaya 1994; Ganjian 2007).

Pyrrophyta has the highest cell abundance in spring, which may be the environmental conditions during the spring lead to the growth. In the summer, cyanophyta had the highest density, which could be attributed to the increase in water temperature as seen in the Blanes Bay (Hense and Beckmann 2006), and the highest cell abundant of diatoms in the winter and autumn. Different researchers have reported that temperature was an important factor in fluctuations in the composition of phytoplankton and changes phytoplankton population 


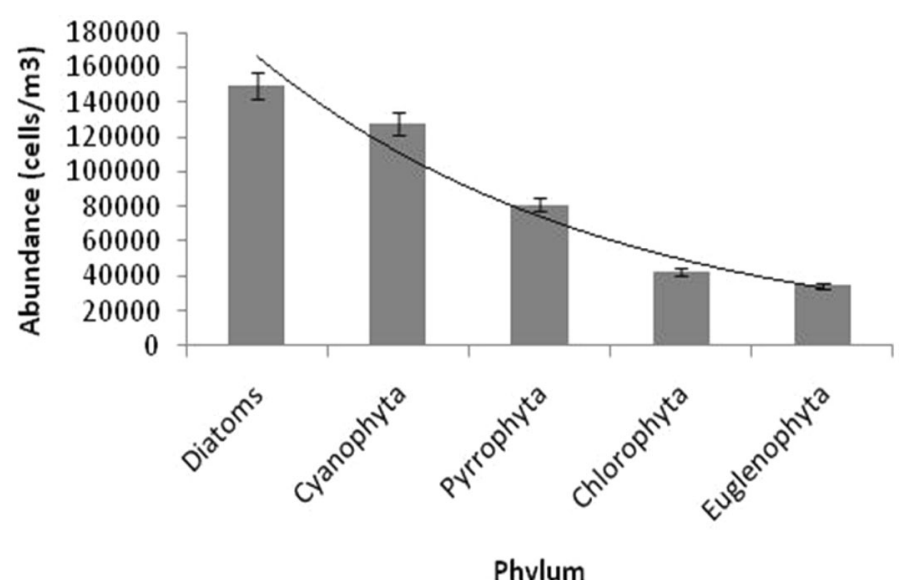

Fig. 6 The annual distribution changes in the average cell abundance $\left(\right.$ cells $/ \mathrm{m}^{3}$ ) of phytoplankton phyla

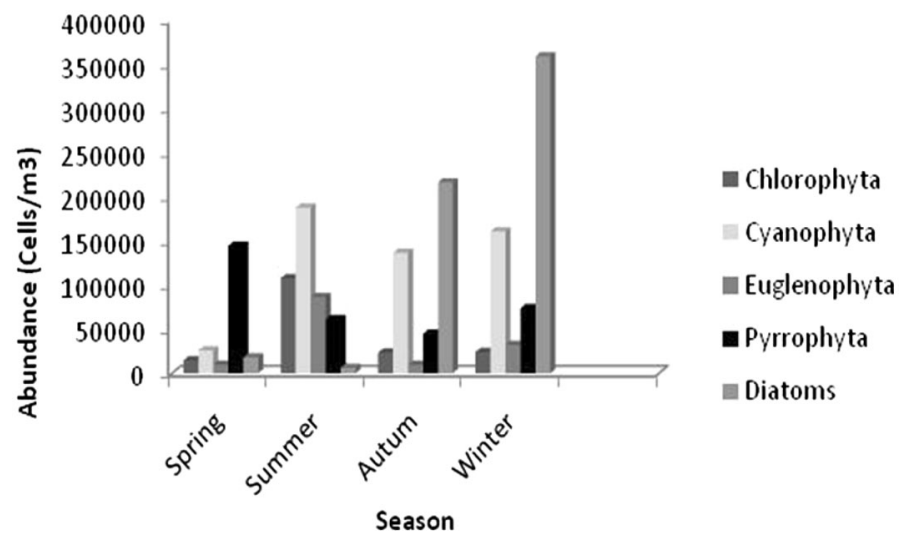

Fig. 7 Comparison between densities of different phyla of phytoplankton in different seasons

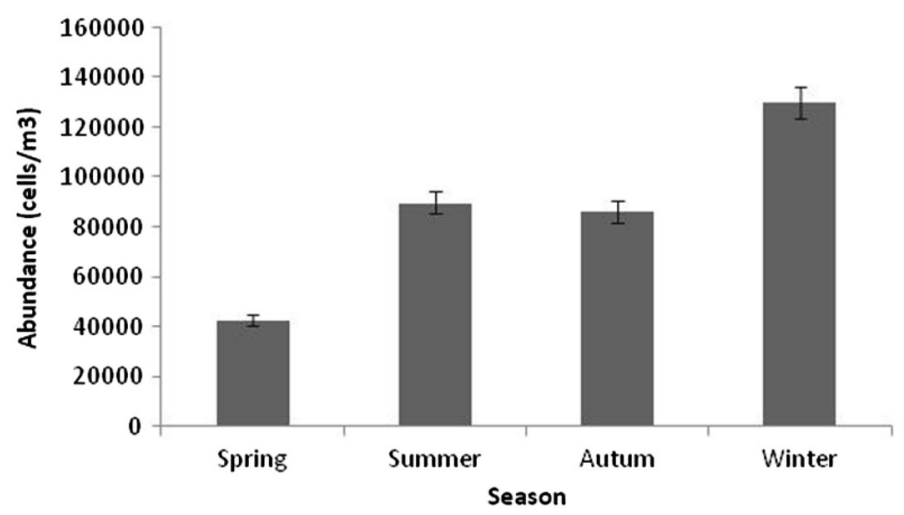

Fig. 8 The total average of cell abundance $\left(\right.$ cells $\left./ \mathrm{m}^{3}\right)$ of phytoplankton in different seasons

with changing season and temperature differences, and diatoms were dominant species of the cold season in the Caspian Sea (Kideys et al. 2005; Resende et al. 2007; Bagheri et al. 2012). Compared to the previous findings, an increasing trend was observed in phytoplankton cell density. For example, the annual average density of phytoplankton was reported by Kosarev and Yablonskaya (1994) $\left(1.4 \times 10^{4}\right.$ cell L $\left.^{-1}\right)$, Kideys et al. (2005) $\left(4.0 \times 10^{4}\right.$ cell $\left.\mathrm{L}^{-1}\right)$, and Bagheri et al. $(2010)\left(2.10 \times 10^{5}-3.90 \times 10^{5}\right.$ cell $\left.\mathrm{L}^{-1}\right)$ with the highest 
abundance of Pyrrophyta (51-56\%) and Cyanophyta (22-29\%). Dominant phytoplankton species were Prorocentrum cordatum of Pyrrophyta and Oscillatoria sp. of Cyanobacteria, and the present study $\left(43.55 \times 10^{4}\right.$ cell $/ \mathrm{m}^{3}$ ) showed that there is a large variation in the density of phytoplankton in the Caspian Sea.

Also in the same sea, Ozgur et al. 2010 showed that the annual average density of phytoplankton in the south Black Sea Samsun Bay was less than $0.01 \times 10^{6}$ in February 2003 and reached maximum value of $1.20 \times 10^{6}$ cells $\mathrm{L}^{-1}$ in July.

This survey showed that diatoms such as Thalassionema nitzschiodes and pyrrophyte Exuviaella cordata and cyanophyte Oscillatoria limosa were prevalent in Phytoplankton populations at the site.

Bagheri et al. (2014) showed that diatom communities were represented by a few dominant species such as D. fragilissimus and $T$. nitzschioides in the system.

In a previous research by Nasrollahzadeh Saravi et al. (2014) identified phytoplankton species, Exuviaella cordata, Oscillatoria sp., Pseudonitzschia seriata, Thalassionema nitzschioides, Cerataulina pelagica and Chrysochromulina sp. indicated the most abundance. Cyanophyta are living organisms from the natura ecosystem. Pointed out that warm (more than $20^{\circ} \mathrm{C}$ ), calm and stratified water in summer are desirable for the growth of Oscillatoria sp. (Chorus and Bartram 1999), Oscillatoria sp. is one of dwelling species of the Caspian Sea. However, this high abundance of the species in 2009 was introduced in the category of "harmful and potential bloom species "in the region.

In this study, dominant species had the highest density at a depth of $10 \mathrm{~m}$. Also, the highest cell abundance of phytoplankton was observed in Bandar coasts transect. In this transect, due to the presence of breakwater, some species have come from the Anzali Wetland toward the sea, which increased the density of the phytoplankton compared with other transects.

In the Caspian Sea, various factors such as hydrological regimes, climate anomalies, increasing detergents, increasing the flow of fresh water through rivers, and increasing the level of nutrients due to the discharge of industrial and agricultural waste can affect the diversity and density of phytoplankton in coastal water of Caspian Sea (Kideys et al. 2008; Bilio and Niermann 2004).

Bagheri et al. (2014) theorized that hydro-biological changes in the Caspian Sea, Black Sea, and Baltic Sea during the 1990s and 2000s could be definitely correlated with climatical and hydrological characters.

\section{Conclusion}

There was no evidence of bloom in Bandar-e Anzali in southwestern of Caspian Sea. Although, species were observed that have a potential for blooming on this site, which should be controlled before reaching the bloom. As mentioned, increasing the detergents, fertilizers and nutrients resulting from industrial and agricultural sewages to the wetlands and coastal water of Caspian Sea and increase in deforestation are important elements for nutrient sources, and increase in these materials is an important factor in increasing the production of phytoplankton. The two top points were observed in the summer and winter. The top points were formed with $O$. limosa and T. nitzschiodes species from the phyla of Cyanophyta (29\%) and diatom (34\%), respectively. Opportunistic reproduction, toxin production and harmful characteristics of dominant species in the two seasons are the evidences of the under stress features of the Caspian Sea, which should be considered for environmental solutions.

Acknowledgements We are grateful to Laboratory of Department of the Environment, Marine Environment Division, Guilan, Iran. We wish to thank Mr. Armoodly in Fisheries Department of Iran. The Department of Marine Biology, Science and Research Branch, Islamic Azad University, Tehran, Iran is also gratefully acknowledged.

Open Access This article is distributed under the terms of the Creative Commons Attribution 4.0 International License (http:// creativecommons.org/licenses/by/4.0/), which permits unrestricted use, distribution, and reproduction in any medium, provided you give appropriate credit to the original author(s) and the source, provide a link to the Creative Commons license, and indicate if changes were made. 


\section{References}

APHA (2005) Standard method for the examination of water and wastes water. American Publication Health Association, Washington, pp 10.1-10.47

Bagheri S, Mashhor M, Makaremi M, Mirzajani AR, Babaei H, Negarestan H, Wan-Maznah WO (2010) Distribution and composition of phytoplankton in the southwestern Caspian Sea during 2001-2002, a comparison with previous surveys. World J Fish Mar Sci 2(5):416-426

Bagheri S, Mashhor M, Turkoglu M, Makaremi M, Babaei A (2012) Temporal distribution of phytoplankton in the south-western Caspian Sea during 2009-2010: a comparison with previous surveys. J Mar Biol Assoc UK 92(6):1243-1255. https://doi.org/ $10.1017 /$ S0025315412000094

Bagheri S, Turkoglu M, Abedini A (2014) Phytoplankton and nutrient variations in the Iranian waters of the Caspian Sea (Guilan region) during 2003-2004. Turk J Fish Aquat Sci 14:231-245. https://doi.org/10.4194/1303-2712-v14_1_25

Bat L, Sezgin M, Satilmis HH, Sahin F, Üstün F, Birinci-Özdemir Z, Gökkurt Baki O (2011) Biological diversity of the Turkish Black Sea Coast. Turk J Fish Aquat Sci 11:683-692. https://doi.org/10.4194/1303-2712-v11_4_04/trjfas

Bilio M, Niermann U (2004) Is the comb jelly to blame for it all? Mnemiopsis leidyi and the ecologial concerns about the Caspian Sea. Mar Ecol Prog Ser 269:173-183. https://doi.org/10.3354/meps269173

Boni AD (1925) Phytoplankton. Translated by Mohammad Reza Rahimi Bashar 2001 Publication of Green Code, p 218

Castro P, Huber ME (1991) Marine biology. McGraw-Hill Higher Education, New York, p 496

Chorus I, Bartram J (1999) Toxic cyanobacteria in water, A guide to their public health consequences, monitoring and management. E \& FN Son, London, p 416P

Dumont HJ (1998) The Caspian Lake: history, biota, structure, and function. J Limnol Oceanogr 43:44-52

Falkowski PG, Raven JA (1997) Aquatic photosynthesis. Blackwell Science, Malden

Ganjian A (2007) Distribution, abundance and biomass of phytoplankton in the southern part of Caspian Sea (in Iranian waters), Thesis, Master of Science. School of Biological Sciences University Sciences Malaysia, p 144

Ganjian A, Wan Maznah WO, Khairun Y, Najafpour Sh, Najafpour GhD, Roohi A (2009) The assessment of biological indices for classification of water quality in southern part of Caspian Sea. World Appl Sci J 7(9):1097-1104

Ganjian A, Wan Maznah WO, Yahya K, Fazli H, Vahedi M, Roohi A, Farabi SMV (2010) Seasonal and regional distribution of phytoplankton in the southern part of the Caspian Sea. Iran J Fish Sci 9(3):382-401

Ghasemov, Bagherov (1983) The biology of Caspian Sea translated by Fathola pour fisheries research center of Gilan, p 184

Griffith MB, Hill BH, McCormick FH, Kauffman PR, Herlihy AT, Selle AR (2002) Comparative application of indices of biotic integrity based on periphyton, macroinvertebrata, and fish to southern rocky mountain streams. Ecol Ind 5(2):117-136

Grigorovich IA, Therriault TW, MacIsaac HJ (2003) History of aquatic invertebrate invasions in the Caspian Sea. Biol Invasions 5:103-115

Hense I, Beckmann A (2006) Towards a model of cyanobacteria life cycle-effects of growing and resting stages on bloom formation of N2-fixing species. In: Ecological modeling, I95. Elsevier, pp 205-218

Izaguirre I, Ofarrell I, Tell G (2001) Variation in phytoplankton composition and limnological features in a water-water ecotone of lower Parana Basin (Argentina). Freshw Biol 46:63-74

Kasymov A (2000) Methods of monitoring in Caspian Sea. In: QAPP-POLIQRAF, p 57

Kideys AE, Soydemir N, Eker E, Vladymyrov V, Soloviev D, Melin F (2005) Phytoplankton distribution in the Caspian Sea during March 2001. Hydrobiologia 543:159-168

Kideys AE, Roohi A, Eker-Develi E, Melin F, Beare D (2008) Increased chlorophyll levels in the southern Caspian Sea following an invasion of jellyfish. Res Lett Ecol 1-5

Kosarev AN, Yablonskaya EA (1994) The Caspian Sea. SPB Academic Publication, Moscow, p 259P

Marques JC, Salas F, Patricio J, Teixeiva H, Neto JM (2009) Ecological indicators for coastal and estuarine environmental assessment, a user's guide. WIT Press, University of Coimbra, Portugal, p 208. ISBN: 978-1845642099

Nasrollahzadeh Saravi H, Makhlough A, Eslami F, Leroy Suzanne AG (2014) Features of phytoplankton community in the southern Caspian Sea, a decade after the invasion of Mnemiopsis leidyi. Iran J Fish Sci 13(1):145-167

Newell GE (1977) Marine plankton. Hutchinson Co., London, p 320

Ozgur B, Arif G, Tufan K (2010) Temporal variations of phytoplankton in relation to eutrophication in Samsun Bay, Southern Black Sea. Turk J Fish Aquat Sci 10:363-372. https://doi.org/10.4194/trjfas

Prescott GW (1962) Algae of the western great lakes area. Brown company Publishing, Iowa, pp 1-933

Proshkina-Lavrenko AI, Makarova IV (1968) Plankton algae of the Caspian Sea. Leningrad, Nauka, p 287 (in Russian)

Resende P, Azeiteiro UM, Goncalves F, Pereira MJ (2007) Distribution and ecological preferences of diatoms and dinoflagellata in the west Iberian coasta zone (North Portugal). Acta Oecol 32:224-235. https://doi.org/10.1016/j.actao.2007.05.004

Salmanov MA (1987) The role of Microflora and phytoplankton in production process. Translated by Abolghasem shariati the science and industrial fishery centers in Mirza Kochagkhan, Rasht, p 349

Tiffany LH, Britton LE (1971) The algae of Illinois. Hanfer Publishing Company, New York, pp 1-407

Vereshchaka AL, Anokhina LL (2014) Composition and dynamics of the Black Sea Benthopelagic plankton and its contribution to the near-shore plankton communities. PLoS One 9(6):e99595. https://doi.org/10.1371/journal.pone.0099595

Vollenweider AR (1974) A manual on methods for measuring primary production in aquatic environmental. Blackwell Scientific publication Oxford, London, p 423

\section{Publisher's Note}

Springer Nature remains neutral with regard to jurisdictional claims in published maps and institutional affiliations. 